\title{
Dipole Model to Predict the Rectangular Defect on Ferromagnetic Pipe
}

\author{
V. Suresh ${ }^{1 *}$ and A. Abudhair ${ }^{2}$ \\ ${ }^{1}$ National Engineering College, Department of Electronics and Instrumentation Engineering, Kovilpatti, Tamilnadu, India \\ ${ }^{2}$ Vel Tech Multitech Dr. Rangarajan Dr. Sakunthala Engineering College, Department of Electrical and Electronics Engineering, \\ Chennai, India
}

(Received 31 March 2016, Received in final form 9 June 2016, Accepted 23 June 2016)

\begin{abstract}
Dipole model based analytical expression is proposed to estimate the length and depth of the rectangular defect on ferromagnetic pipe. Among the three leakage profiles of Magnetic Flux Leakage (MFL), radial and axial leakage profiles are considered in this work. Permeability variation of the specimen is ignored by considering the flux density as close to saturation level of the inspected specimen. Comparing the profile of both the components, radial leakage profile furnishes the better estimation of defect parameter. This is evident from the results of error percentage of length and depth of the defect. Normalized pattern of the proposed analytical model radial leakage profile is good agreement with the experimentally obtained profile support the performance of proposed expression.
\end{abstract}

Keywords : Magnetic Flux Leakage, dipole model, radial leakage profile, axial leakage profile

\section{Introduction}

Magnetic Flux Leakage (MFL) technique is well established one to detect the defect on ferromagnetic specimen. Ferromagnetic material is used to fabricate steam generator tubes, tank floor, rail road and etc. The periodical inspection is required to prevent accident due to the defect on the ferromagnetic specimen. In MFL technique, inspected specimen is magnetized to near saturation level and hence any metal anomalies cause a magnetic leakage field. Sensor which is placed in the inspection tool measures the leakage profile. Two methods of the MFL testing are practiced vis-à-vis forward and inverse problem method. Calculation of the defects from the known defect profile is referred as forward problem [1]. Analytical and finite model based approaches afford solutions to the forward problem. Former approach has minimum computation time when compared with the latter [2]. Analytical approach has to provide the information to develop the MFL tools [3]. Three dimension of the MFL field due to the defect on ferromagnetic specimen has been derived from the concept of dipolar theory [4].

Optimum magnetic saturation of the inspected specimen

(C)The Korean Magnetics Society. All rights reserved.

*Corresponding author: Tel: +91-94436-95768

Fax: +91-4632-232749, e-mail: vseie@nec.edu.in is essential to obtain the good MFL signal from the defect [5]. An analytical expression for radial component of MFL of a right angular parallel piped surface crack has been proposed. The maximum percentage of error for this method is $12 \%$ for depth and $30 \%$ for width of the crack [6]. Asymptotic method based analytical approach has been proposed to detect the defined shape of the surface defect. The radial component of the profile was obtained from the axial component by means of the Hilbert transform and also concluded that the density of defect induced magnetic charges has been directly related to the surface shape [7]. Xin Dai et al. proposed an analytical model for radial-flux permanent-magnet eddy-current couplings while considering the complex boundary conditions. For validation purpose they have carried out the finite element analysis [8].

The effect of discontinuity orientation and sensor scanning direction on MFL signal has been studied. Higher sensitivity of the leakage profile has been attained when the scanning direction is perpendicular to the discontinuity orientation [9]. Suresh et al. developed an analytical model for radial leakage profile of cylindrical hole defect on ferromagnetic pipe. Different lift-off studies also carried out and concluded that the $0.1 \mathrm{~mm}$ distance between the specimen and sensor is the optimum lift-off to detect the defect [10]. Numerical and experimental studies have been carried out by Reza Khalaj Aminesh et al. to charac- 
terize the rectangular shape defect on the ferromagnetic material using tangential component of the leakage profile. Normalization of the leakage profile has been carried to compare the numerical and experimental results [11]. COMSOL based numerical studies have been carried out for a magnetizer arrangement with dimension mentioned in this paper to produce the uniform magnetic field on outer wall on the inspected specimen while the probe is placed inside the pipe [12].

In this paper, analytical model for radial and axial leakage profile of the rectangular notch is proposed using the dipole theory. Permeability variation of the specimen is ignored by considering the flux density as close to saturation level, as consideration of permeability would yield more complexity in analytical model. MATLAB coding is developed for both analytical models. Comparison studies of the radial and axial component are made. Length and Depth of the defects are estimated directly from the leakage profile of the both models and observed that the error percentage of the radial leakage profile is very minimum compare to the axial leakage profile. The pattern of the proposed radial leakage profile is compared with the experimental result.

\section{Analytical Model}

In analytical approach, the radial leakage profile of the rectangular shape defect is formulated mathematically by dipole method. Figure 1 shows the dipole representation of rectangular defect with the positive and negative charge density based on the applied magnetic field. The defect has length ' $l$ ', width ' $w$ ' and depth 'b'.

The magnetic field generated at a distance $\mathrm{R}$ due to the magnetic polarity is given by,

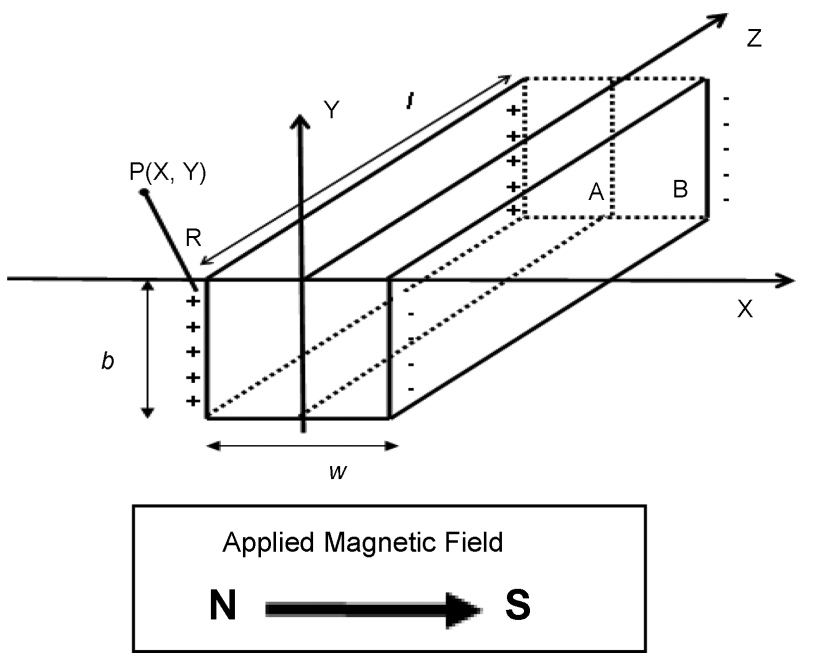

Fig. 1. Dipolar representation of Rectangular defect.

$$
d B=\frac{d p}{\left(4 \pi R^{3}\right)} \vec{R}
$$

The radial leakage field can be derived from the first principle of Maxwell equation.

$$
\begin{aligned}
& B_{y}{ }^{+}=\frac{\sigma}{4 \pi}\left[\frac{1}{\sqrt{(x-w)^{2}+y^{2}}}-\frac{1}{\sqrt{(x-w)^{2}+(y+b)^{2}}}\right] \\
& B_{y}{ }^{-}=\frac{-\sigma}{4 \pi}\left[\frac{1}{\sqrt{(x-w)^{2}+y^{2}}}-\frac{1}{\sqrt{(x-w)^{2}+(y+b)^{2}}}\right]
\end{aligned}
$$

The total radial leakage field can be computed by the equation (4).

$$
B_{y}=B_{y}^{+}+B_{y}^{-}
$$

The axial leakage field can be derived from the first principle of Maxwell equation.

$$
\begin{gathered}
B_{x}^{+}=\frac{\sigma(x-w)}{4 \pi}\left[\begin{array}{l}
\frac{y+b}{(x-w)^{2} \sqrt{(y+b)^{2}+(x-w)^{2}}} \\
-\frac{y}{(x-w)^{2} \sqrt{y^{2}+(x-w)^{2}}}
\end{array}\right] \\
B_{x}^{-}=\frac{\sigma(x+w)}{4 \pi}\left[\begin{array}{l}
\frac{y+b}{(x+w)^{2} \sqrt{(y+b)^{2}+(x+w)^{2}}} \\
-\frac{y}{(x+w)^{2} \sqrt{y^{2}+(x+w)^{2}}}
\end{array}\right]
\end{gathered}
$$

The total axial leakage field can be computed by the equation (7).

$$
B_{x}=B_{x}^{+}+B_{x}^{-}
$$

MATLAB 14.0 software was employed to obtain the solution for the above equation. The results of analytical model for various dimensions of the rectangular defects are shown in the section 4 .

\section{Experimental Studies}

This section describes the experimental studies carried out at our NDE laboratory. An artificial rectangular defect of $4 \mathrm{~mm}$ length, $1 \mathrm{~mm}$ width and $2.3 \mathrm{~mm}$ of depth was machined on the outer diameter of the ferromagnetic steam generator (SG) pipe by electro-discharge machining (EDM) process. The length of the tube is $1000 \mathrm{~mm}$, the inner diameter is $12.6 \mathrm{~mm}$ and the wall thickness is 2.3 $\mathrm{mm}$.

Agilent make Programmable DC regulated power supply 


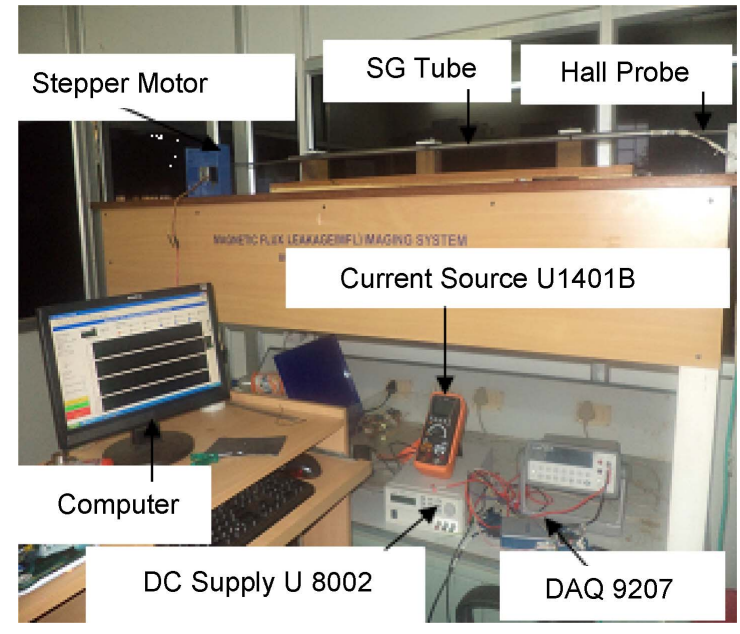

Fig. 2. (Color online) Experimental setup.

(U8002A) was used for power source to the magnetizer coil. Cheng yang Technologies, Germany, manufactured Indium Stibnite (InSb) semiconductor Hall sensor (CYSH12AF) was employed to detect the leakage profile. Hall sensor has a better resolution than search coil and also the range of linearity is higher compare to other kind of magnetic sensors [12, 13]. Agilent make Multi Function Calibrator/Meter (U1401B) was utilized for current source to the Hall sensor. Hall sensor output was connected to the personal computer by means of National Instruments supplied 24 bit resolution DAQ 9207. National Instruments (NI) make, MID-7602- 2 Axis Stepper Motor Drive and PCI 7352 Motion Controller were utilized to achieve the performance of the automated probe pulling mechanism and the programs are developed using LabVIEW2011.

Figure 2 shows the arrangement of the experimental

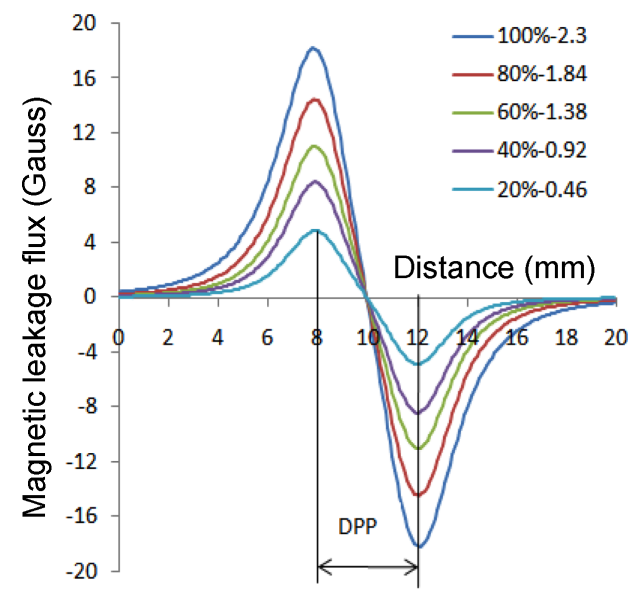

(a) setup along with the automatic probe pulling mechanism. This system helps to perform forward and reverse linear scanning of SG tube with a resolution of $1 \mathrm{~mm} / \mathrm{step}$. The linear movement is achieved by means of sprocket - chain mechanism which is controlled by NI micro-step stepper motor, motion control card - PCI 7352 and MID driver -9602 .

Uniform magnetization of the inspected specimen is achieved through the bobbin type of probe arrangement. Dimension of the probe is as follows; both end of the probe side referred as guider region length is $10 \mathrm{~mm}$ and coil wounded area is $30 \mathrm{~mm}$. Sensor is placed on the centre of the coil wounded area to sense the radial leakage profile of the magnetic field. Uniform magnetic field through the bobbin coil on the inspected specimen is achieved through the four ampere current which is passed on coil with 130 numbers wound on the bobbin. Supply current to the hall sensor is $5 \mathrm{~mA}$ to obtain the linear output of leakage profile. The above said probe arrangement is placed inside the inspected pipe yet its sense the defect on outer wall. Automatic probe pulling mechanism scanned the entire region of the pipe when the probe reached the defect, Hall sensor in the probe sense the leakage field and send the signal to the data acquisition card. Raw data is processed using LabVIEW2011.

\section{Results and Discussion}

Figure 3 shows output of the radial and axial leakage profile obtained analytically for rectangular defect. The dimension of the defect is as follows, length four $\mathrm{mm}$, width one $\mathrm{mm}$ and different $\mathrm{mm}$ of depth. Distance between the peak to peak (DPP) of the radial leakage profile shows the exact length of the defect.

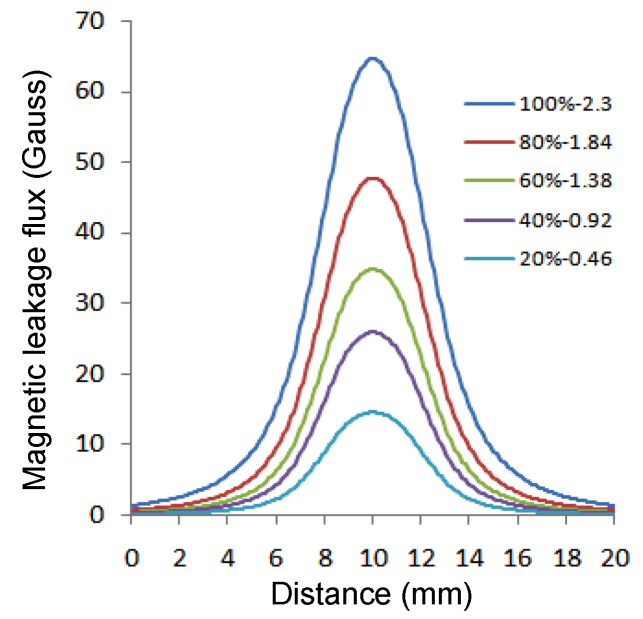

(b)

Fig. 3. (Color online) (a) Radial, (b) Axial leakage profile for different depth dimension of rectangular notch. 


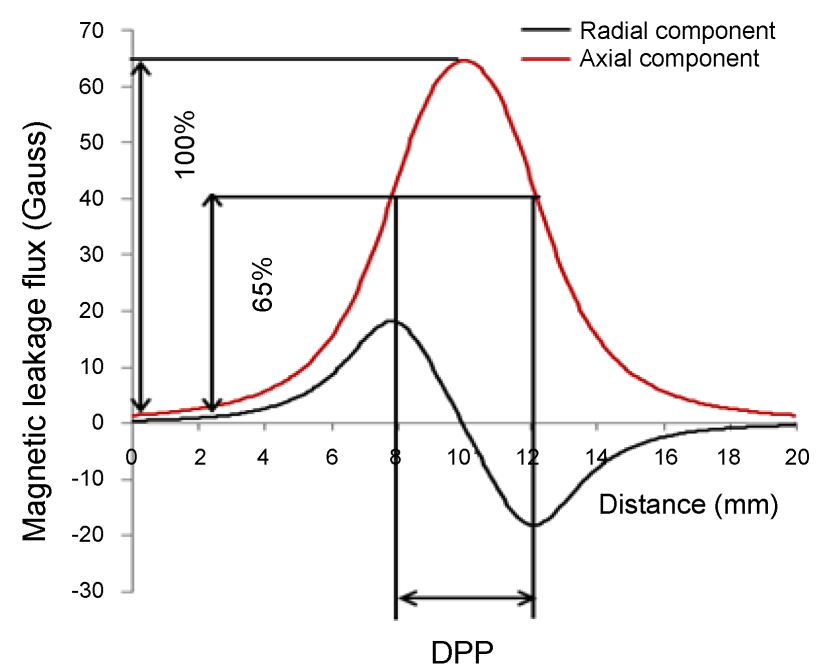

Fig. 4. (Color online) Radial and Axial leakage profile for $100 \%$ depth of the rectangular notch.

Figure 4 shows the super imposed of analytically obtained output of radial and axial leakage profile for the above mentioned dimension of the rectangular defect. DPP location of radial leakage is extended to the axial leakage profile and it is observed that the magnitude of the $65 \%$ of axial profile distance represents the exact length of the defect as shown in Fig. 4.

Table 1 shows the error percentage of length estimation in axial and radial profile. It is observed that the radial leakage profile estimate the accurate length of the defect even in minimum depth of the defect but in contrast axial leakage profile is failed to estimate the defect of length in minimum depth dimension.

Depth estimations have been carried out using radial and axial leakage profile. From the Fig. 3, it is observed that the depth of the defect is responsible for peak amplitude of the leakage profile. Polynomial expression is derived based on the curve which fit between depth and its corresponding amplitude. Polynomial expression for depth of the defect is expressed (8) for axial leakage profile. The peak amplitude of axial leakage profile is
Table 2. Error percentage of depth estimation in axial and radial profile.

\begin{tabular}{ccccc}
\hline \hline $\begin{array}{c}\text { Actual } \\
\text { Depth } \\
(\mathrm{mm})\end{array}$ & $\begin{array}{c}\text { Axial profile } \\
\text { Estimated } \\
\text { depth } \\
(\mathrm{mm})\end{array}$ & $\begin{array}{c}\text { Radial profile } \\
\text { Estimated } \\
\text { depth } \\
(\mathrm{mm})\end{array}$ & $\begin{array}{c}\text { Axial } \\
\text { profile } \\
\text { Error (\%) }\end{array}$ & $\begin{array}{c}\text { Radial } \\
\text { profile } \\
\text { Error }(\%)\end{array}$ \\
\hline 2.3 & 2.3 & 2.3 & 0 & 0 \\
1.84 & 1.82 & 1.85 & 1.08 & -0.54 \\
1.38 & 1.35 & 1.34 & 2.17 & 2.89 \\
0.92 & 0.97 & 0.94 & -5.43 & -2.17 \\
0.46 & 0.43 & 0.45 & 6.52 & 2.17 \\
\hline
\end{tabular}

represented as $B_{x}$.

$$
\text { Depth }=-0.0003 B_{x}^{2}+0.0597 B_{x}-0.3930
$$

Polynomial expression for depth of the defect is expressed (9) for radial leakage profile. The peak amplitude of radial leakage profile is represented by $B_{y}$.

$$
\text { Depth }=-0.00273 B_{y}{ }^{2}+0.0204 B_{y}-0.0345
$$

Table 2 shows the percentage of error in depth estimation using axial and radial profile. Maximum percentage of error in depth estimation from the axial profile and radial profile is as follows $\pm 6.52 \%, \pm 2.89 \%$. From the result it is observed that radial profile has minimum error compared to the axial profile.

In experimental study the Hall sensor (CYSH12AF) is employed to sense the radial leakage profile. Sensor output is connected to the computer by using National Instruments supplied 24 bit resolution DAQ 9207 and the noise is removed using LabVIEW. Experimental and analytical output is normalized with respect to their corresponding maximum amplitude. Normalization doesn't change the position of the corresponding maximum amplitudes.

Figure 5 shows the normalized radial leakage profile of analytical and experimental output for the dimension of the $4 \mathrm{~mm}$ length, $1 \mathrm{~mm}$ width and $2.3 \mathrm{~mm}$ depth of the rectangular defect. Normalized pattern of the proposed

\begin{tabular}{|c|c|c|c|c|c|c|c|}
\hline \multirow[b]{2}{*}{$\begin{array}{l}\text { Depth } \\
(\mathrm{mm})\end{array}$} & \multicolumn{3}{|c|}{ Axial profile } & \multirow{2}{*}{$\begin{array}{c}\text { Radial profile } \\
\text { Estimated } \\
\text { Length DPP } \\
(\mathrm{mm})\end{array}$} & \multirow[b]{2}{*}{$\begin{array}{l}\text { Actual Length } \\
\qquad(\mathrm{mm})\end{array}$} & \multirow[b]{2}{*}{$\begin{array}{c}\text { Axial } \\
\text { profile } \\
\text { Error }(\%)\end{array}$} & \multirow[b]{2}{*}{$\begin{array}{c}\text { Radial } \\
\text { profile } \\
\text { Error (\%) }\end{array}$} \\
\hline & $\begin{array}{c}100 \% \\
\text { Amplitude } \\
\text { (Gauss) }\end{array}$ & $\begin{array}{c}65 \% \\
\text { Amplitude } \\
\text { (Gauss) }\end{array}$ & $\begin{array}{l}\text { Estimated } \\
\text { Length } \\
(\mathrm{mm})\end{array}$ & & & & \\
\hline 2.3 & 65 & 42.25 & 4 & 4 & 4 & 0 & 0 \\
\hline 1.84 & 48 & 31.20 & 4 & 4 & 4 & 0 & 0 \\
\hline 0.92 & 26 & 16.90 & 4.1 & 4 & 4 & -2.5 & 0 \\
\hline 0.46 & 15 & 9.75 & 4.15 & 4 & 4 & -3.75 & 0 \\
\hline
\end{tabular}

Table 1. Error percentage of length estimation in axial and radial profile. 


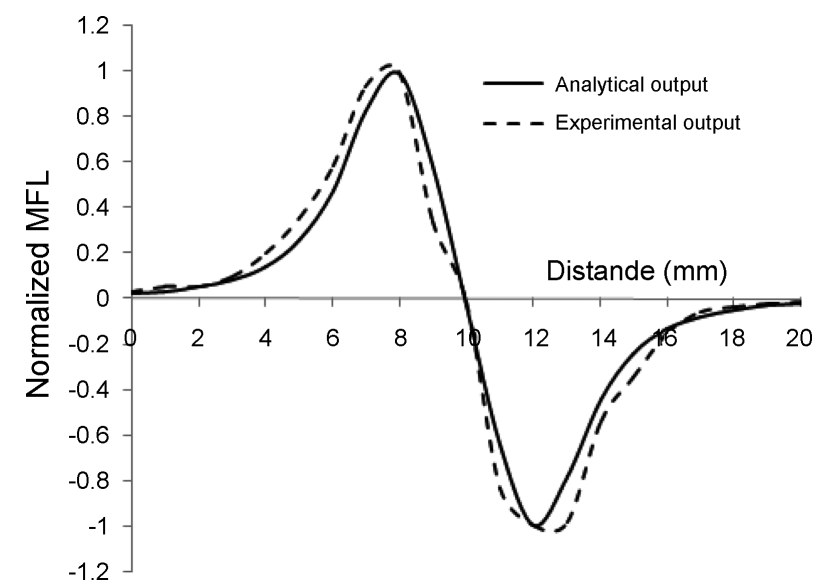

Fig. 5. Radial leakage profile of Analytical and Experimental results.

analytical model leakage profile is good agreement with the experimentally obtained profile support the performance of proposed expression.

\section{Conclusion}

In this paper, simple analytical model for Radial and Axial leakage profile of MFL for rectangular defect is proposed. Length and Depth of the defects were estimated from the result of proposed analytical model. Radial leakage profile has estimated the actual length with $0 \%$ error. But in the case of axial leakage profile the result has $\pm 3.75 \%$ error. Depth estimation using radial and axial leakage profile has $\pm 2.89 \%$ and $\pm 6.52 \%$ error respectively. When compared with axial leakage profile, the radial leakage profile provides better estimation of defect dimension. Experimental result for the radial leakage profile also supports the analytical output. The proposed radial leakage profile analytical expression is simple and it can be used for reconstruction of defect in inverse MFL problem.

\section{References}

[1] S. M. Dutta, F. H. Ghorbel, and R. K. Stanley, IEEE Trans. Magn. 45, 1966 (2009).

[2] C. Mandache and L. Clapham, Phys. D: Appl. Phys. 36, 2427 (2003).

[3] G. Kopp and H. Willems, NDT \& E International 55, 75 (2013).

[4] S. M. Dutta, F. H. Ghorbel, and R. K. Stanley, IEEE Trans. Magn. 45, 1959 (2009).

[5] G. S. Park, P. W. Jung, and Y. W. Rho, J. Magn. 6, 31 (2001).

[6] D. Minkov, J. Lee, and T. Shoji, J. Magn. Magn. Mater. 217, 207 (2000).

[7] S. Lukyanets, A. Snarskii, M. Shamonin, and V. Bakaev, NDT\&E International 36, 51 (2003).

[8] X. Dai, Q. Liang, C. Ren, J. Cao, J. Mo, and S. Wang, J. Magn. 20, 273 (2015).

[9] J. Wu, Y. Sun, Y. Kang, and Y. Yang, IEEE Trans. Magn. 51, 6200207 (2015).

[10] V. Suresh and A. Abudhahir, Measurement Sci. Rev. 16, 8 (2016).

[11] R. K. Amineh, N. K. Nikolova, J. P. Reilly, and J. R. Hare, IEEE Trans. Magn. 44, 516 (2008).

[12] P. Karuppasamy, A. Abudhahir, M. Prabhakaran, S. Thirunavukkarasu, B. P. C. Rao, and T. Jayakumar, J. Nondestructive Evalu. 35, 5 (2015).

[13] D.-G. Park, M. B. Kishore, J. Y. Kim, L. J. Jacobs, and D. H. Lee, J. Magn. 21, 57 (2016).

[14] CYSH 12 AF (InSb) Hall effect element, www.cy-sensors.com, Chen Yang Technologies GmbH \& Co. KG. 